\title{
Correspondence
}

\section{Effectiveness increases with integration in primary care}

It is good to read of the work of Dr de Silva and his colleagues in South Tyneside. ${ }^{1}$ They are seeing people at an impressive hit rate and providing a service which people like. As in Gnosall, the model being used takes advantage of primary care settings. I think the difference is that we provide expertise within primary care, with a view to a potential three tiers (primary, secondary, tertiary), ${ }^{2}$ whereas de Silva is describing a secondary tier outreach. The advantage of Gnosall, which has been demonstrated now over nearly 9 years, is that continuity of support and integration of care are facilitated and sustained. ${ }^{3,4}$ Great stuff though: people are catching on!

1 De Silva P. What about effectiveness? [eLetter] BJPsych Bull 2015; 11 February.

2 Jolley D, Greaves I, Greaves N, Greening L. Three tiers for a comprehensive regional memory service. J Dementia Care 2010; 18: 26-9.

3 Greaves I, Greaves N, Walker E, Greening L, Benbow S, Jolley D. Gnosall primary care memory clinic: eldercare facilitator role description and development. Dementia 2013; 12: doi: 10.1177/1471301213497737.

4 Clark M, Moreland N, Greaves I, Greaves N, Jolley D. Putting personalisation and integration into practice in primary care. J Integrated Care 2013; 21: 105-20.

David Jolley is Consultant Psychiatrist, University of Manchester, email: david.jolley@manchester.ac.uk, Susan M. Benbow is Professor of Psychiatry at the University of Chester and visiting Consultant Psychiatrist at Gnosall Medical Centre, and lan Greaves is General Practitioner at Gnosall Medical Centre, Staffordshire.

doi: $10.1192 / p b .39 .4 .205$

\section{CMHTs provide follow-up for patients with dementia and behavioural and psychological symptoms of dementia in both service models}

David Jolley makes a valuable point about the need for ongoing support for patients receiving a dementia diagnosis and we agree that all patients deserve such input. ${ }^{1}$ We disagree that our patients are failed by either the memory clinic combined with the community mental health team (CMHT) service or the traditional CMHT service described in our paper, ${ }^{2}$ as both services have good relationships with general practitioners (GPs), who can refer rapidly into the CMHT arms of both services as any behavioural and psychological symptoms of dementia arise. Jolley criticises post-diagnostic signposting to the third sector as leaving patients and their relatives adrift. But these post-diagnostic services have been specifically commissioned from a third sector organisation and not from secondary care, which has the disadvantage of not being part of psychiatric services but is an acceptable, 'non-medicalised' service that can be accessed at any time. The study of the third sector organisation was not within the remit of our paper.

We did not comment specifically on referral rates and this study examined only a small part of the service in these trusts, so we are not certain where the figure of 5 per 1000 that Jolley quotes is derived from. In fact, the services have quite different levels of staffing (both medical and non-medical) and there are other memory services in the region provided by neurologists and geriatricians as well (not examined in our paper), so we had specifically not commented on referral rates in total but only on these small patches within the service.

Both services have changed considerably since 2011 and some service improvements have been inspired specifically by this evaluation. Both services now ensure multidisciplinary follow-up for all patients (where patients and their carers are advised verbally and in writing how to contact various local services according to their needs in the future) and the memory-clinic-based service has cut down on some paper assessment tools. More therapy treatments are offered in both services. Other changes include the introduction of nurse specialists to assist in the memory-clinic-based service. The CMHT service continues to offer consultant medical domiciliary diagnostic assessment, with prescribing now done by GPs from the outset and initial monitoring and post-diagnostic support provided through the CMHT.

We are concerned about the lack of research into these services, rapidly changing across the country. The introduction of new models should be accompanied by robust independent evaluation and evidence of sustained benefit over a sufficient period to prove worth. Multiple innovations in the context of constantly reorganised health and social care systems have no evidence base to justify them from the perspective of frail older people where continuity has been demonstrated to be highly valued.

1 Jolley D. Heads in the sand may leave old age psychiatry looking foolish and vulnerable. Commentary on ... A memory clinic v. traditional community mental health team service. BJPsych Bull 2015; 39: 12-4.

2 Rubinsztein JS, van Rensburg MJ, Al-Salihy Z, Girling D, Lafortune L, Radhakrishnan $M$, et al. A memory clinic v. traditional community mental health team service: comparison of costs and quality. BJPsych Bull 2015; 39: 6-11.

Judy S. Rubinsztein, Consultant in Old Age Psychiatry, Norfolk and Suffolk NHS Foundation Trust, email: judy.rubinsztein@nsft.nhs.uk; Deborah Girling, Consultant in Old Age Psychiatry, Cambridgeshire and Peterborough NHS Foundation Trust; Carol Brayne, Director, Cambridge Institute of Public Health, Department of Public Health and Primary Care, University of Cambridge.

doi: 10.1192/pb.39.4.205a

\section{Thoughts on the development of liaison psychiatry services in London}

The clear strength of Naidu et al's paper ${ }^{1}$ is its attempt to map the development of liaison services in London over the past 8 years. The authors have also appraised the various models of liaison services. It was interesting to see which models have been adopted in Greater London as well as the variations that exist, including the absence of a liaison service in one trust.

When we were reviewing policy documents, ${ }^{2}$ it has caught our attention that recommended staff numbers have not changed since they were first proposed by the Royal College of Psychiatrists in 2007. The context for this observation is the continuing reduction in acute bed numbers as well as increased recognition of the need to promptly identify and treat psychiatric comorbidities in acute settings. 